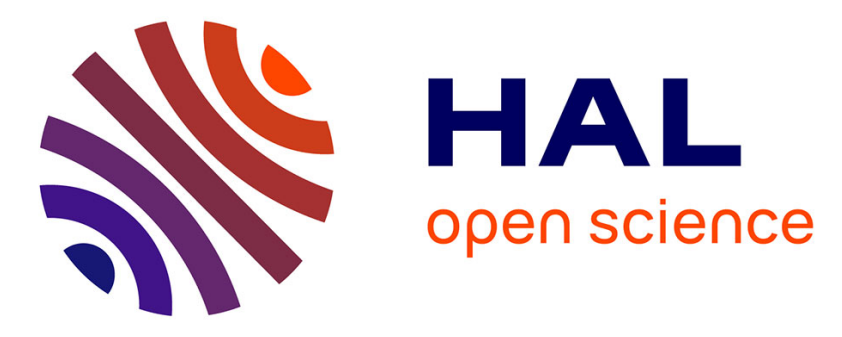

\title{
Wireless Coupling of Conducting Polymer Actuators with Light Emission
}

\author{
Bhavana Gupta, Mariana C Afonso, Lin Zhang, Cédric Ayela, Patrick
} Garrigue, Bertrand Goudeau, Alexander Kuhn

\section{To cite this version:}

Bhavana Gupta, Mariana C Afonso, Lin Zhang, Cédric Ayela, Patrick Garrigue, et al.. Wireless Coupling of Conducting Polymer Actuators with Light Emission. ChemPhysChem, 2019, 20 (7), pp.941-945. 10.1002/cphc.201900116 . hal-02101543

\section{HAL Id: hal-02101543 \\ https://hal.science/hal-02101543}

Submitted on 16 Apr 2019

HAL is a multi-disciplinary open access archive for the deposit and dissemination of scientific research documents, whether they are published or not. The documents may come from teaching and research institutions in France or abroad, or from public or private research centers.
L'archive ouverte pluridisciplinaire HAL, est destinée au dépôt et à la diffusion de documents scientifiques de niveau recherche, publiés ou non, émanant des établissements d'enseignement et de recherche français ou étrangers, des laboratoires publics ou privés. 


\title{
Wireless coupling of conducting polymer actuators with light
} emission

\author{
Bhavana Gupta $^{[a]}$, Mariana C. Afonso ${ }^{[a]}$, Lin Zhang ${ }^{[a]}$, Cedric Ayela ${ }^{[b]}$, Patrick Garrigue ${ }^{[a]}$, Bertrand \\ Goudeau $^{[a]}$, Alexander Kuhn*[a]
}

\begin{abstract}
Combining the actuation of conducting polymers with additional functionalities is an interesting fundamental scientific challenge and increases their application potential. Here we demonstrate the possibility of direct integration of a miniaturized light emitting diode (LED) in a polypyrrole (PPy) matrix in order to achieve simultaneous wireless actuation and light emission. A light emitting diode is used as a part of an electroactive surface on which electrochemical polymerization allows direct incorporation of the electronic device into the polymer. The resulting free-standing polymer/LED hybrid can be addressed by bipolar electrochemistry to trigger simultaneously oxidation and reduction reactions at its opposite extremities, leading to a controlled deformation and an electron flow through the integrated LED. Such a dual response in the form of actuation and light emission opens up interesting perspectives in the field of microrobotics.
\end{abstract}

\section{Introduction}

Smart materials responding to one or more external stimuli in a controlled and reproducible way is an emerging topic of research due to their potential use in different fields ranging from aeronautic, automotive and electronic devices, to drug delivery. ${ }^{1-}$ ${ }^{6}$ Smart materials play a significant role in the field of robotics and micromachines, as the functionality of one part depends on the stimulus from another part. ${ }^{7-11}$ Generally, when designing smart materials and devices which respond to various stimuli or provide more than one response for a given stimulus, an integration of several entities is required. ${ }^{12,13}$ For example, Torres Alonso et al recently developed smart textiles, based on fibers endowed with touch-sensing and light-emitting functionalities. ${ }^{14}$ However, combing several functions in a hybrid device is in most cases a difficult task. Furthermore, in order to get a response, smart materials normally need to be connected directly with some sophisticated instrumentation, either to provide stimuli or to read the output signal. The equivalent of an $\mathrm{IQ}$ can be defined for smart materials and depends on three important factors: responsiveness, agility and complexity. ${ }^{5}$ Thus, materials providing a visual output signal such as light, colour change or mechanical deformation fall in the category of smart materials with a higher IQ.

[a] Dr. B. Gupta, M. Afonso, Dr. L. Zhang, P. Garriague, Dr. B. Goudeau, Prof. A. Kuhn

Univ. Bordeaux, ISM UMR CNRS 5255, Bordeaux INP, ENSCBP, 16 avenue Pey Berland, 33607 Pessac, France

E-mail:kuhn@enscbp.fr

[b] Dr. C. Ayela

Univ. Bordeaux, IMS, CNRS, UMR 5218, Bordeaux INP, ENSCBP,

F-33405 Talence, France

Supporting information for this article is given via a link at the end of the document
Conducting polymers belong to one of the various categories of smart materials which can generate an electromechanical output upon receiving an electrical stimulus. ${ }^{15}$ Recently, we were able to use polypyrrole (PPy) to generate mechanical output upon electrochemical stimulation without any direct connection to a power supply, thus the actuation is truly wireless. ${ }^{16-18}$ A polymer strip undergoes both swelling and shrinking simultaneously due to the intrinsic asymmetry generated in the polymer as a result of the global electric field present during a bipolar electrochemical experiment. The electric field polarizes the polymer in such a way that one extremity participates in an oxidation reaction, while the other one is undergoing a reduction process. Either color change or light emission induced by bipolar electrochemistry has already been reported with different conducting polymers. ${ }^{19-21}$ However, a major challenge in this context is to obtain both light and actuation simultaneously to increase the application potential and the smartness of conducting polymers.

In general, breaking the symmetry based on bipolar electrochemistry in terms of shape, composition or other physico-chemical features of conducting and semiconducting objects is already very well known. Prominent examples comprise color change ${ }^{20,22}$, site selective deposition ${ }^{23-27}$, exfoliation $^{28}$, motion $^{29-33}$, light emission ${ }^{34,35}$ and chemical read out, $^{18,36,37}$ etc. Bipolar electrochemistry has also been reported to drive electronic devices ${ }^{38}$, leading for example to light emitting e-swimmers. ${ }^{39}$ Here we propose to combine the interesting features of PPy and those of a LED, enabling in fine a controlled light emission and simultaneous actuation. Such a single body incorporation increases the visual output signal in response to an electric field as a stimulus, similar to interesting alternative literature approaches ${ }^{40,41}$, and adds on to the smart behavior of the conducting polymers for the development of multifunctional miniaturized systems.

\section{Results and Discussion}

During the wireless powering of the hybrid device, the chemical reactions occurring at its extremities are the key factors for simultaneous light emission and actuation. The general concept for the light generation is similar to what has been reported for light emitting e-swimmers ${ }^{39}$, however with an important variation of the involved chemical reactions. Since the conducting polymer is itself electrochemically active ${ }^{15,42,43}$, PPy reduction and oxidation can occur at its two extremities. The overall mechanism of the simultaneous actuation and light emission is illustrated in Scheme 1. When applying a global electric field by the feeder electrodes, the hybrid bipolar electrode, which is fixed in the center of the cell, is polarized in such a way that at the $\delta^{+}$ extremity PPy is oxidized, and at the $\delta$ - side PPy reduction occurs. If the negative pole of the incorporated LED is oriented towards the $\delta^{+}$side of the polymer, the two extremities of the 
PPy start bending in opposite directions, accompanied by light emission when the LED threshold voltage is reached. If the negative pole of the LED is oriented towards the $\delta$ - extremity of the polymer, light emission is not possible due to the blocking effect of the semiconductor junction. The bending direction of PPy depends on its surface state. Its two faces intrinsically don't have the same roughness and porosity because during the electrosynthesis one face is orientated towards the solution whereas the other one is in contact with the smooth electrode. As a consequence, ion insertion and expulsion takes place with different efficiencies at the two faces, leading to unequal swelling and shrinking, and thus directional bending, analog to a classic bilaminar structure. ${ }^{16}$ In order to bend downwards at the $\delta$ extremity and upwards at $\delta^{+}$extremity during the first application of the electric field, the polymer needs to be placed with the rough interface up. At the $\delta$ end, more cations $\left(\mathrm{Li}^{+}\right)$ move into the polymer on the rough face than on the smooth face, thus causing bending from the rough towards the smooth face. In contrast, at the $\delta^{+}$extremity, more cations leave through the rough face and therefore the polymer bends in the upward direction. For longer polarization times and also when changing the polarity of the feeder electrodes, the direction of bending reverses because of the influence of the counter anion (dodecylbenzene sulfonate) present inside the oxidized polymer. The $\delta$ extremity starts showing upward bending due to preferential anion expulsion from the rough side and vice versa.

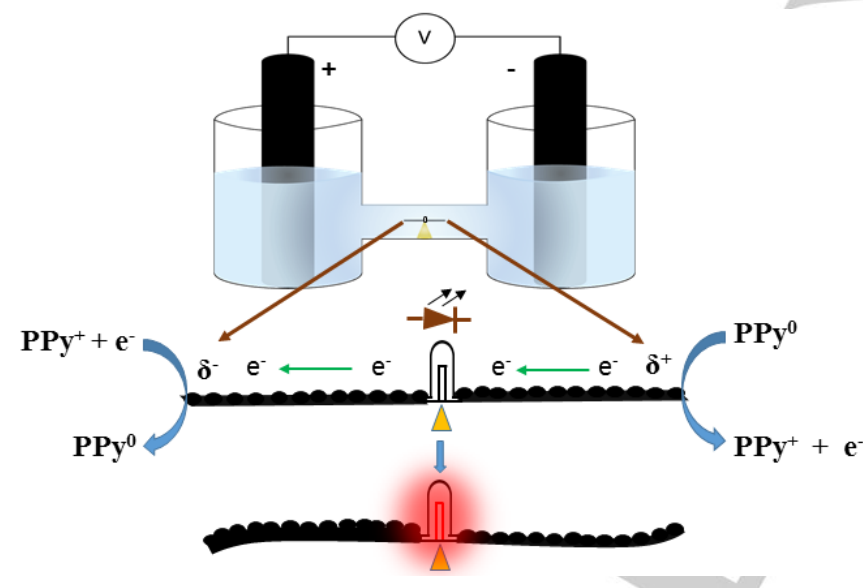

Scheme 1. Schematic illustration of the simultaneous actuation and light emission from the hybrid PPy/LED device during the first instance of polarization. Green arrows indicate the direction of electron flow. $\delta^{+}$and $\delta$ symbolize the polarization of the PPy bipolar electrode during exposure to the electric field. The diode symbol illustrates the direction of current flow during forward bias.

Since actuation and light emission are solely due to the electrochemical reactions at the two extremity of the polymer, the oxidation state of PPy at the two ends plays an important role.

For the purpose of light emission, a miniaturized LED with four connecting gold pads is used (Figure 1A). Two pads are for powering a red light LED and the other two for green light. As for every diode, current can only flow in one direction i.e. from the positive pole to the negative pole. However, for a given applied voltage bias $(2 \mathrm{~V})$, the current flowing through the red light emitter is about one order of magnitude higher compared to the green LED and the first one also has a lower threshold voltage (Figure 1B).
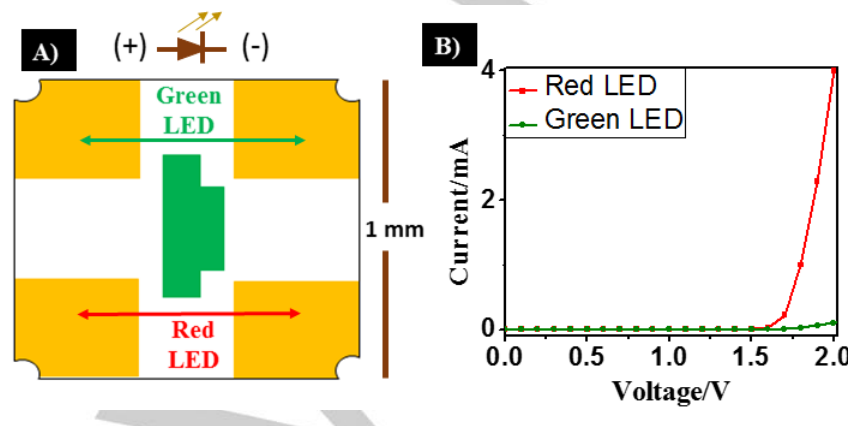

Figure 1. A) Illustration of the miniaturized LED with the diode symbol to show the direction of electron flow during forward bias and B) Comparative I-V curves for the green and red LED.

For connecting the polymer with the electronic device, we used the electrochemical growth of PPy directly on the connecting pads of the LED which is placed on the surface of a gold coated glass slide. In this case, the polymer can only deposit on the four gold connectors and the gold coated glass electrode. Adhesive tape and varnish is placed on certain parts of the LED to avoid polymer growth and short circuits (Figure 2A and 2B).
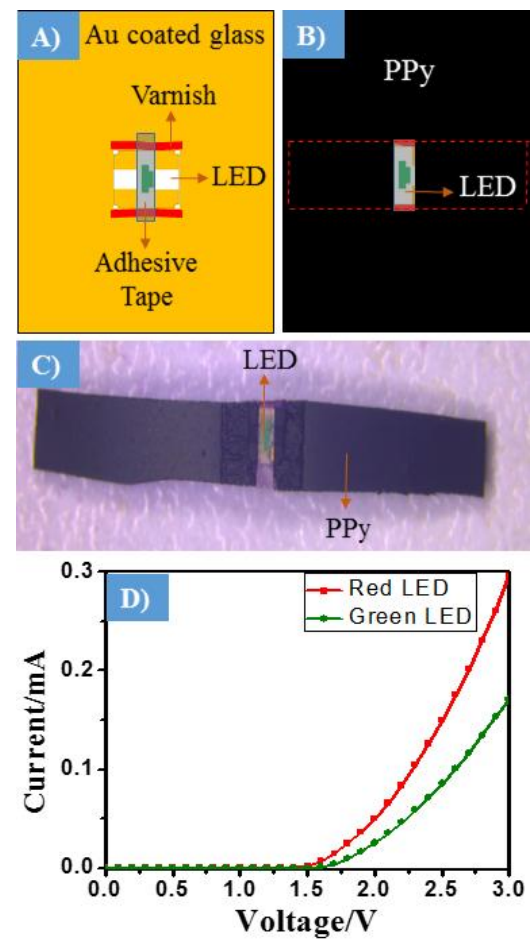
Figure 2. A) Scheme of the LED $(1 \mathrm{~mm} \times 1 \mathrm{~mm})$ position on the surface of a gold coated glass slide before polymerization. Thick red lines indicate a varnish deposit to prevent electropolymerisation in these areas. Adhesive tape (light green rectangle) is positioned on top of the LED to avoid overgrowth of PPy and shortcircuiting B) Scheme of the LED embedded in a polypyrrole film after polymerization; the red dotted line shows where the PPy is going to be cut in order to get the final integrated device C) Photograph of the final hybrid actuator D) I-V curves of the green and red LED after integration into the polypyrrole strip when the voltage bias is directly applied via the polymer.

The pairs of gold pads for the red and green LED are connected by the deposited PPy. Thus, when applying a bias voltage via the common PPy strip, only red light is visible, because the red LED provides a more favorable path for charge transport (Figure 2D). However, when compared to Figure 1B, the overall current decreases, probably due to the contact resistance at the junction between PPy and gold. During bipolar electrochemistry, only red light emission is expected during forward bias along with electromechanical deformation. To increase the mechanical strength of the junction, a thin layer of transparent varnish is deposited onto the junction. The resulting free standing hybrid film is then used for wireless actuation and simultaneous light emission.

The electrosynthesis of the conducting polymer occurs by oxidative polymerization, thus the as-synthesized polymer backbone contains positive charges, counter balanced with anions. For the first bipolar actuation experiments, the PPy is kept in a fully oxidized state. The threshold electric field for light emission is around $2 \mathrm{~V} / \mathrm{cm}$, however at this driving force only very little deformation of PPy is observed. As expected, only red light emission occurs based on the electrical property of the integrated LED (Figure 1B and 2D). When increasing the electric field to $4 \mathrm{~V} / \mathrm{cm}$, the $\delta$ extremity of the polymer starts bending (Figure $3 \mathrm{~b}$ and $\mathrm{c}$ ).

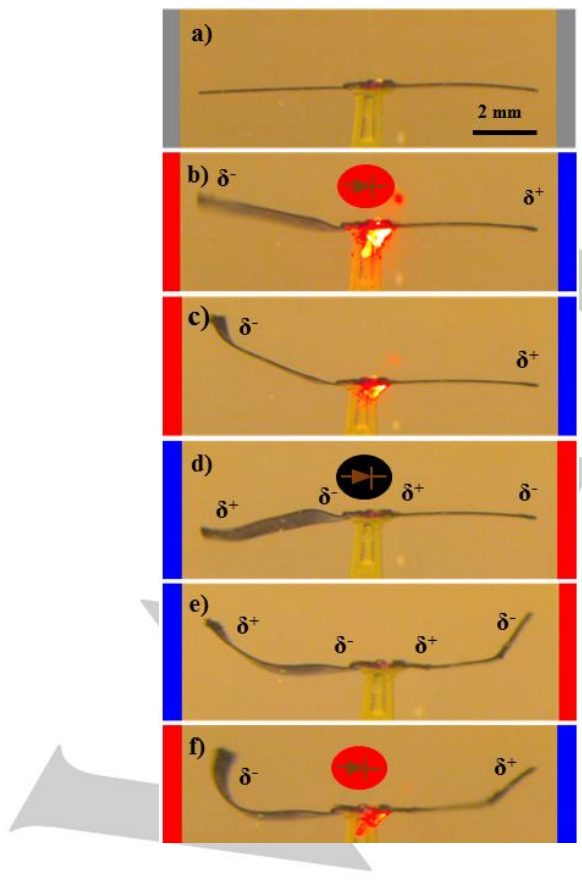

Figure 3. Different situations of wireless bipolar actuation as a function of the switching electric field with a fully oxidized PPy film mounted on an insulating pillar in $0.5 \mathrm{M} \mathrm{LiClO}_{4}$ a) before applying the field, b) $\left.13^{\text {th }} \mathrm{c}\right) 13^{\text {th }}$ (after $5 \mathrm{sec}$ ) d) $18^{\text {th }}$ e) $26^{\text {th }}$ and f) $27^{\text {st }}$ cycle of switching the electric field. The LED symbol illustrates forward and blocking bias. Red: positive feeder electrode; Blue: negative feeder electrode; Gray: feeder electrodes without applying electric field. The electric field is $4 \mathrm{~V} / \mathrm{cm}$ and $7 \mathrm{~V} / \mathrm{cm}$ for b), c) and d), e), f) respectively. $\delta^{+}$and $\delta^{-}$indicate the polarization of the PPy film at the two extremities.

In the beginning of the actuation, light emission is very intense however, with increasing actuation the light intensity decreases. This can be explained by the fact that during bending the effective length of the bipolar electrode decreases, as does the related polarization and consequently also the light intensity. The polymer extremity with $\delta^{+}$polarity doesn't show any electromechanical actuation at $4 \mathrm{~V} / \mathrm{cm}$. This is expected since PPy is already in its completely oxidized state and therefore only solvent oxidation can occur at this pole. During the reverse bias, the LED will be in its blocking state, which means that the initial polymer strip must now be considered as being composed of two electrically independent bipolar electrodes with half of the initial length and separated by a very large resistance (Figure 3 d). Under these conditions, there are four polarization points (Figure $3 \mathrm{~d}$ and $3 \mathrm{e}$ ) in contrast to two polarization points during forward polarization (Figure 3f). Due to the effect of the reverse blocking bias, the length of PPy is shorter, which means that a higher electric field is needed to electromechanically activate the two half-length bipolar electrodes. Therefore, by increasing the electric field, the PPy extremity which was electromechanically inactive during the forward bias also starts bending (Figure 3e). Due to the two times shorter length, the electric field required for bending needs to be roughly two times higher $(7 \mathrm{~V} / \mathrm{cm})$ compared to a polymer without the blocking LED. ${ }^{16}$

To make PPy electrochemically and electromechanically active at both extremities at smaller electric fields i.e. $4 \mathrm{~V} / \mathrm{cm}$, the initial PPy strip needs to be in a partially reduced state. Interestingly, a partially reduced strip of PPy demonstrates bipolar actuation at both extremities from the very first bipolar cycle with an electric field of $4 \mathrm{~V} / \mathrm{cm}$ as shown in Figure $4 \mathrm{~b}$ and $\mathrm{c}$.

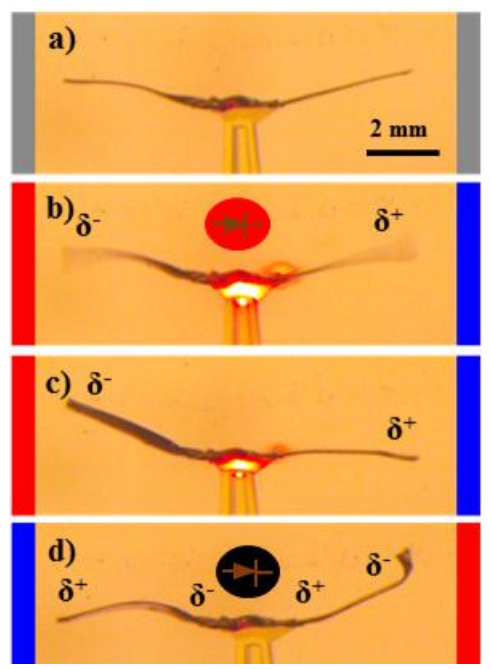


Figure 4. Bipolar actuation of a partially reduced PPy film as a function of the switching electric field in $0.5 \mathrm{M} \mathrm{LiClO}_{4}$ a) before applying the field, b) $3^{\text {rd }}$ c) $3^{\text {rd }}$ (after $5 \mathrm{sec}$ ) and d) $4^{\text {th }}$ cycle of switching the electric field. The electric field is 4 $\mathrm{V} / \mathrm{cm}$.

The light intensity is also comparatively higher than in the case when the polymer is in its fully oxidized state, probably due to a more efficient charge transport from one end to the other. During the reverse blocking bias, the oxidation and reduction reactions at the four polarization points of the separated two bipolar electrodes still allow reversible actuation at $4 \mathrm{~V} / \mathrm{cm}$ for many cycles, accompanied by light emission (Video 1 supporting information). Compared to such a partly reduced PPy, the electrochemical reactions are not inversed symmetrically at the two extremities of a fully oxidized PPy. Such a break of symmetry can be the ingredient for generating directional motion. In order to demonstrate this effect, we place the hybrid PPy/LED object at the bottom of the bipolar cell with the rough side downwards in order to induce a crawling motion. ${ }^{17}$ As soon as a field of $7 \mathrm{~V} / \mathrm{cm}$ is applied with a forward bias for the LED, red light emission is observed (Figure 5 b). During subsequent polarity switching, the polymer strip adapts an asymmetric shape due to the unequal oxidation states of the two extremities. During the period with four polarization points under reverse blocking bias, the more electroactive bipolar half-electrode develops a pushing effect with respect to the other bipolar halfelectrode. This results in a directional motion of the object comparable to a crawler. (Video 2 supporting information).

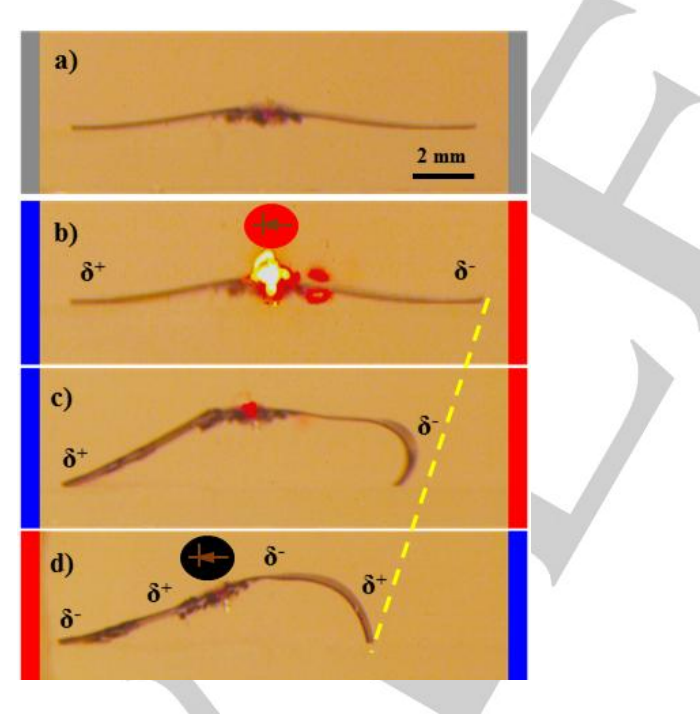

Figure 5. Snapshots of wireless crawling with light emission using a fully oxidized PPy strip in $0.5 \mathrm{M} \mathrm{LiClO}_{4}$ a) before applying the electric field, b) $1^{\text {st }} \mathrm{c}$ ) $15^{\text {th }}$ d) $16^{\text {th }}$ cycle of switching the electric field. The electric field is $7 \mathrm{~V} / \mathrm{cm}$. The dotted line indicates the total displacement during bipolar crawling.

\section{Conclusions}

We successfully integrated a miniaturized electronic device into a free-standing PPy film by directly electropolymerizing pyrrole at the LED interface. The strong interaction between the two physical entities is responsive to electrical stimuli, leading simultaneously to electromechanical actuation and light emission. This is the first set of proof-of-concept experiments where two completely different optically recordable phenomena are triggered in parallel and in a wireless way. The effect is based on the asymmetric electrochemical reactions occurring at the two extremities of the PPy strip. This asymmetry is also used for inducing directional motion of the hybrid object. In the future, such devices should open the door for the development of various smart micro-machines and robotic structures.

\section{Experimental Section}

\section{Embedding of a Light Emitting Diode in polypyrrole}

The LED (PICOLED ${ }^{\text {TM }}$-DUO, ROHM Co. Ltd) with $1 \mathrm{~mm} \times 1 \mathrm{~mm}$ dimensions was attached to the surface of a gold coated glass slide in sequential steps. In the first step, double-sided adhesive tape was fixed on the surface of the gold coated glass slide. The LED was fixed in the center of the tape. After fixing, gold sputtering was carried out. Before gold sputtering the light emitting region is protected by precisely positioning under the microscope a small piece of adhesive parafilm on the top of the light emitter. After sputtering, the parafilm protection was removed. Finally, the last and most crucial step is to apply a varnish casting at the two sides of the LED and placing adhesive tape onto the centre of the light emitting part of the LED (Figure 2A). The obtained device is then ready for electrochemical polymerization.

For embedding the LED in a polypyrrole film, a monomeric solution of pyrrole (0.2 M) (Sigma Aldrich) was dissolved in Milli Q water along with $0.25 \mathrm{M}$ dodecyl benzene sulfonate (Sigma Aldrich). After complete dissolution of both components, the gold coated glass plates of $2.5 \times 1.5$ $\mathrm{cm}$ with the immobilized LED (working electrode) was fixed parallel to another unmodified gold electrode (counter electrode) of same dimensions in a beaker containing $10 \mathrm{ml}$ of precursor solution and $\mathrm{Ag} / \mathrm{AgCl}$ as a reference electrode. A fixed current of $3.6 \mathrm{~mA}$ was applied for 5400 s for the polymerization of pyrrole. In order to get partially reduced PPy, a potential of $-0.7 \mathrm{~V}$ was applied for $5 \mathrm{~min}$ at the end of the polymerisation process. After completion of polymerization, the polymer coated working electrode was washed with distilled water. Then the polymer, together with the double-sided tape, was slowly peeled off the gold coated glass plate with the help of a sharp knife. It was kept between two glass slides overnight in order to prevent bending. Finally, the flat and free-standing film device was used for bipolar actuation and light emission.

\section{Bipolar actuation and light emission}

For bipolar actuation and light emission, the hybrid LED-polypyrrole strip of $12 \times 1 \mathrm{~mm}$ size was placed in the centre of the bipolar cell, either fixed on a support pillar or free at the bottom of the cell, for the actuation and crawling experiments respectively. Two feeder graphite electrodes were positioned $5 \mathrm{~cm}$ apart on both sides of the film in such a way that each

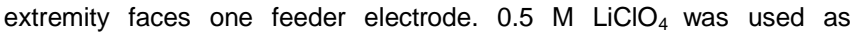
electrolyte to provide sufficient ionic conductivity for the bipolar electrochemistry. Electric fields of different amplitudes were then applied for various times. Actuation and light emission was recorded using a macroscope (LEICA Z16 APO) in video mode. 


\section{Acknowledgements}

This study has received financial support from the French State in the framework of the "Investments for the future" Program, IdEx Bordeaux, reference ANR-10-IDEX-03-02. M.C.A. is grateful for support from CAPES and CNPq (Brazil). A.K. acknowledges financial support from the Institut Universitaire de France. The work has also received funding from the European Research Council (ERC) under the European Union's Horizon 2020 research and innovation program (grant agreement $n^{\circ}$ 741251, ERC Advanced grant ELECTRA).

Keywords: Smart materials $\cdot$ conducting polymers $\bullet$ bipolar electrochemistry $\cdot$ wireless actuation $\bullet$ wireless light emission

[1] M. Yoshida, J. Lahann, ACS Nano. 2008, 2, 1101-1107.

[2] Y. Cui, D. Li, H. Bai, Ind. Eng. Chem. Res. 2017, 56, 4887-4897.

[3] W. Xu, K. S. Kwok, D. H. Gracias, Acc. Chem. Res. 2018, 51, 436-444.

[4] P. Chen, Q. Li, S. Grindy, N. Holten-Andersen, J. Am. Chem. Soc. 2015, 137, 11590-11593.

[5] W. Cao, H. H. Cudney, R. Waser, PNAS. 1999, 96, 8330-8331.

[6] A. A. Tregubov, P. I. Nikitin, M. P. Nikitin, Chem. Rev. 2018, 118, 1029410348.

[7] S. Sánchez, L. Soler, J. Katuri, Angew. Chem. Int. Ed. 2015, 54, 1414-1444.

[8] B. Ciui, A. Martin, R. K. Mishra, T. Nakagawa, T. J. Dawkins, M. Lyu, C. Cristea, R. Sandulescu, J. Wang, ACS Sensors. 2018, 3, 2375-2384.

[9] S. Palagi, P. Fischer, Nat. Rev. Mater. 2018, 3, 113-124.

[10] H.-W. Huang, M. S. Sakar, A. J. Petruska, S. Pané, B. J. Nelson, Nat. Commun. 2016, 7, 12263.

[11] J. Wang, Nanomachines: Fundamentals and Applications, Wiley, 2013.

[12] L. D. Zarzar, J. Aizenberg, Acc. Chem. Res. 2014, 47, 530-539.

[13] Y. A. Jodat, P. Lotfi, P. P. S. S. Abadi, J.-Y. Mun, J. Seo, E. A. Shin, S. M. Jung, C. K. Lee, S. R. Shin, ACS Appl. Nano Mater. 2018, 12, 6630 6640

[14] E. Torres Alonso, D. P. Rodrigues, M. Khetani, D.-W. Shin, A. De Sanctis, H. Joulie, I. de Schrijver, A. Baldycheva, H. Alves, A. I. S. Neves, S. Russo, M. F. Craciun, npj Flexible Electron. 2018, 2, 25.

[15] J. G. Martinez, T. F. Otero, E. W. H. Jager, Langmuir. 2014, 30, $3894-$ 3904.

[16] B. Gupta, B. Goudeau, A. Kuhn, Angew. Chem. Int. Ed. 2017, 56, 1418314186.

[17] B. Gupta, B. Goudeau, P. Garrigue, A. Kuhn, Adv. Funct Mater. 2017, 28 , 1705825.

[18] L. Zhang, B. Gupta, B. Goudeau, N. Mano, A. Kuhn, J. Am. Chem. Soc. 2018, 140, 15501-15506.

[19] F. AlTal, J. Gao, J. Am Chem. Soc.. 2018, 140, 9737-9742.

[20] S. Inagi, Y. Ishiguro, M. Atobe, T. Fuchigami, Angew. Chem. Int. Ed. 2010, 49, 10136-10139.

[21] J. Gao, S. Chen, F. AITal, S. Hu, L. Bouffier, G. Wantz, ACS Appl. Mater. Interfaces. 2017, 9, 32405-32410.

[22] Y. Ishiguro, S. Inagi, T. Fuchigami, J. Am. Chem. Soc. 2012, 134, 4034 4036.

[23] L. Koefoed, S. U. Pedersen, K. Daasbjerg, Curr. Opin. Electrochem. 2017, 2, 13-17.

[24] Z. A. Fattah, L. Bouffier, A. Kuhn, Appl. Mater. Today. 2017, 9, 259-265

[25] I. Malytska, C. Mézière, M. Kielar, L. Hirsch, G. Wantz, N. Avarvari, A. Kuhn, L. Bouffier, J. Phys. Chem. C. 2017, 121, 12921-12927.

[26] S. E. Fosdick, K. N. Knust, K. Scida, R. M. Crooks, Angew. Chem. Int. Ed. 2013, 52, 10438-10456.

[27] D. Öhl, Y. U. Kayran, J. R. C. Junqueira, V. Eßmann, T. Bobrowski, W. Schuhmann, Langmuir. 2018, 34, 12293-12301.
[28] D. Bouša, C. C. Mayorga-Martinez, V. Mazánek, Z. Sofer, K. Boušová, M. Pumera, ACS Appl. Mater. Interfaces. 2018, 10, 16861-16866.

[29] G. Loget, A. Kuhn, Nat. Commun. 2011, 2, 535.

[30] W. F. Paxton, P. T. Baker, T. R. Kline, Y. Wang, T. E. Mallouk, A. Sen, J. Am. Chem. Soc. 2006, 128, 14881-14888.

[31] L. Bouffier, D. Zigah, N. Sojic, A. Kuhn in Recent Advances in bipolar electrochemistry, Vol. 27 (Ed. C. G. Zoski. Allen J. Bard), CRC Press, 2017, pp.27-118.

[32] G. Loget, A. Kuhn, J. Am. Chem. Soc. 2010, 132, 15918-15919.

[33] L. Ren, D. Zhou, Z. Mao, P. Xu, T. J. Huang, T. E. Mallouk, ACS Nano. 2017, 11, 10591-10598.

[34] S. Hu, X. Chi, S. Chen, F. AlTal, J. Gao, J. Phys. Chem. C. 2017, 121, 8409-8415.

[35] O. Phuakkong, M. Sentic, H. Li, C. Warakulwit, J. Limtrakul, N. Sojic, A. Kuhn, V. Ravaine, D. Zigah, Langmuir. 2016, 32, 12995-13002.

[36] F. Mavré, R. K. Anand, D. R. Laws, K.-F. Chow, B.-Y. Chang, J. A. Crooks, R. M. Crooks, Anal. Chem. 2010, 82, 8766-8774.

[37] S. Jansod, M. Cuartero, T. Cherubini, E. Bakker, Anal. Chem. 2018, 90 , 6376-6379.

[38] X. Zhang, Q. Zhai, H. Xing, J. Li, E. Wang, ACS Sens. 2017, 2, 320-326.

[39] J. Roche, S. Carrara, J. Sanchez, J. Lannelongue, G. Loget, L. Bouffier, P. Fischer, A. Kuhn, Sci. Rep. 2014, 4, 6705.

[40] S. T. Chang, V. N. Paunov, D. N. Petsev, O. D. Velev, Nat. Mater. 2007, $6,235$.

[41] K. Han, C. W. Shields Iv, O. D. Velev, Adv. Funct. Mater. 2018, 28, 1705953.

[42] A. M. Österholm, J. F. Ponder, J. A. Kerszulis, J. R. Reynolds, ACS Appl. Mater. Interfaces. 2016, 8, 13492-13498.

[43] J. Wang, A. J. Bard, J. Am. Chem. Soc. 2001, 123, 498-499. 


\section{Electrochemical} embedding of a miniaturized LED in a freestanding polypyrrole film allows wireless actuation with simultaneous light emission, triggered by asymmetric electrochemical reactions at the two extremities of the polymer strip.
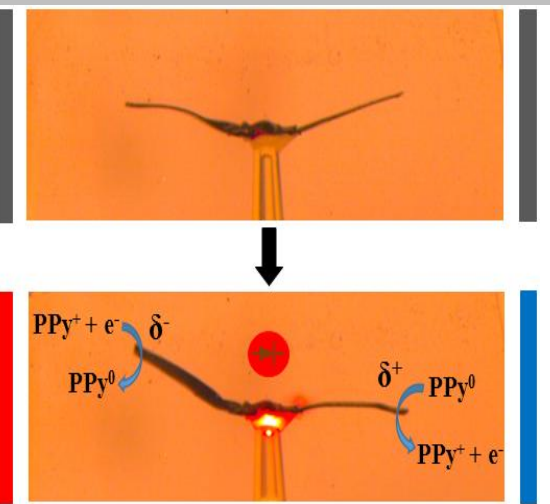

Bhavana Gupta, Mariana C. Afonso, Lin Zhang, Cedric Ayela, Patrick Garrigue, Bertrand Goudeau, Alexander Kuhn *

Page No. - Page No. Wireless coupling of conducting polymer actuators with light emission 